\title{
Spin-density functional study of the organic polymer dimethylaminopyrrole: A realization of the organic periodic Anderson model
}

\author{
Yuji Suwa, ${ }^{1}$ Ryotaro Arita, ${ }^{2}$ Kazuhiko Kuroki, ${ }^{3}$ and Hideo Aoki ${ }^{4}$ \\ ${ }^{1}$ Advanced Research Laboratory, Hitachi, Ltd., Kokubunji, Tokyo 185-8601, Japan \\ ${ }^{2}$ Department of Applied Physics, University of Tokyo, Hongo, Tokyo 113-8656, Japan \\ ${ }^{3}$ Department of Applied Physics and Chemistry, University of Electro-Communications, Chofu, Tokyo 182-8585, Japan \\ ${ }^{4}$ Department of Physics, University of Tokyo, Hongo, Tokyo 113-0033, Japan
}

(Dated: November 8, 2018)

\begin{abstract}
While the periodic Anderson model (PAM) has been recognized as a good model for various heavy f-electron systems, here we design a purely organic polymer whose low-energy physics can be captured by PAM. By means of the spin density functional calculation, we show that polymer of dimethylaminopyrrole is a candidate, where its ground state can indeed be magnetic depending on the doping. We discuss the factors favoring ferromagnetic ground state.

PACS numbers: 71.20.Rv, 71.10.Fd, 75.50.Xx
\end{abstract}

\section{INTRODUCTION}

Realizing organic ferromagnets consisting entirely of non-magnetic elements is a challenging target in

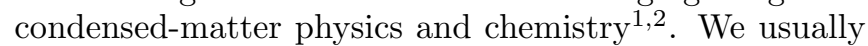
cannot expect itinerant ferromagnetism in organic materials with p-bands, although there are very a few exceptions such as polymethylthiophene $\mathrm{e}^{\underline{3}-\underline{6}}$. Therefore, we need a smart design/strategy to realize purely organic ferromagnets.

Polymers of five membered rings constitute a unique and interesting playground for such materials design, because we can attach a variety of functional groups to the polymers. Indeed, the present authors have proposed that we can realize "flat-band ferromagnetism", originally conceived by Mielke and by Tasaki ${ }^{7.8}$, in aminotriazole with the multi-band Hubbard mode $\underline{1}^{9} \underline{-12}$. While it has been well known that the Hubbard model can have a spin-polarized ground state, it is also well known that the periodic Anderson model, which has been studied extensively especially in the context of the heavy-fermion electron systems, has various magnetic ground states that include a metallic ferromagnet. In the present study, we explore whether a purely organic polymer can be mapped to the periodic Anderson model.

In the course of our attempts, we have found that dimethylaminopyrrole, which can be polymerized comparatively easily $\underline{13}$, is a good candidate for that purpose in that it can accommodate an electronic structure having both narrow and wide bands. By means of spindensity-functional calculations, we shall indeed show that the polymer and oligomers of dimethylaminopyrrole have ferromagnetic $(\mathrm{F})$ or antiferromagnetic $(\mathrm{AF})$ instabilities if holes are sufficiently doped (i.e., more than $1 / 2$ hole per five-membered ring). As for the former instability, we propose that its origin can be explained by the scenario proposed by Batista et al $\underline{\underline{14}}$ While we find that the difference between the energy of the $\mathrm{F}$ state and the $\mathrm{AF}$ states are rather small in this material, we shall finally give a discussion on how the F state can be stabilized.

\section{DIMETHYLAMINOPYRROLE POLYMER}

Let us start with the undoped polymer of dimethylaminopyrrole. Figure 1 $1(\mathrm{a}, \mathrm{b})$ depicts the atomic structure of polydimethylaminopyrrole in a unit cell. We have studied both the spin-unpolarized cases based on the density-functional theory with generalized gradient approximation ${ }^{15,16}$ (GGA-DFT) and the spin-polarized ones based on the spin-density functional theory (GGASDFT). We have used the plane-wave based ultrasoft pseudopotentials $\frac{17.18}{}$ with the energy cutoff at $20.25 \mathrm{Ry}-$ dberg. The convergence criterion of the geometry optimization was that all of the forces acting on each atom were within $1 \times 10^{-3} \mathrm{H} /$ a.u. We have first optimized the structure. While we have started, in our materials design, from a planar configuration of rings, the structureoptimized polydimethylaminopyrrole has dimethylamino bases somewhat twisted out of the plane. This makes the $\pi$-orbital of the nitrogen atom in the amino base lying in the molecular plane, that is, the angle between the $\pi$-orbitals of the nitrogen atom in the amino base and the neighboring nitrogen atom in the five membered ring becomes nearly 90 degrees, leading to a very small $\pi$-bonding between them.

The band structure of undoped polydimethylaminopyrrole is shown in Fig. 1(c). We can see that there exist flat bands (labeled as (B)-(E) and (C)-(F) in Fig. 1(c)), but they lie well below the Fermi energy, unlike the case of polyaminotriazole ${ }^{-}-12$ where the flat band sits around $E_{F}$. In the band structure, a band folding of the quasione-dimensional band is due to the alternating directions of the pyrrole molecules.

Figure 2(A-H) shows the wave functions in the highest four valence (occupied) bands at $\Gamma$ and $\mathrm{X}$ points. The wave functions of the flat bands $(\mathrm{B}, \mathrm{C}, \mathrm{E}$ and $\mathrm{F}$ in Fig. 1(c)) are mixtures of the $\pi$-orbitals of the nitrogen atoms and $\sigma$-orbitals of the carbon atoms, while the other wave functions consist almost entirely of $\pi$-orbitals. We can thus call the flat bands (B)-(E) and (C)-(F) (thick solid lines in Fig. 1(c)) as $\sigma$-bands, and the top valence 

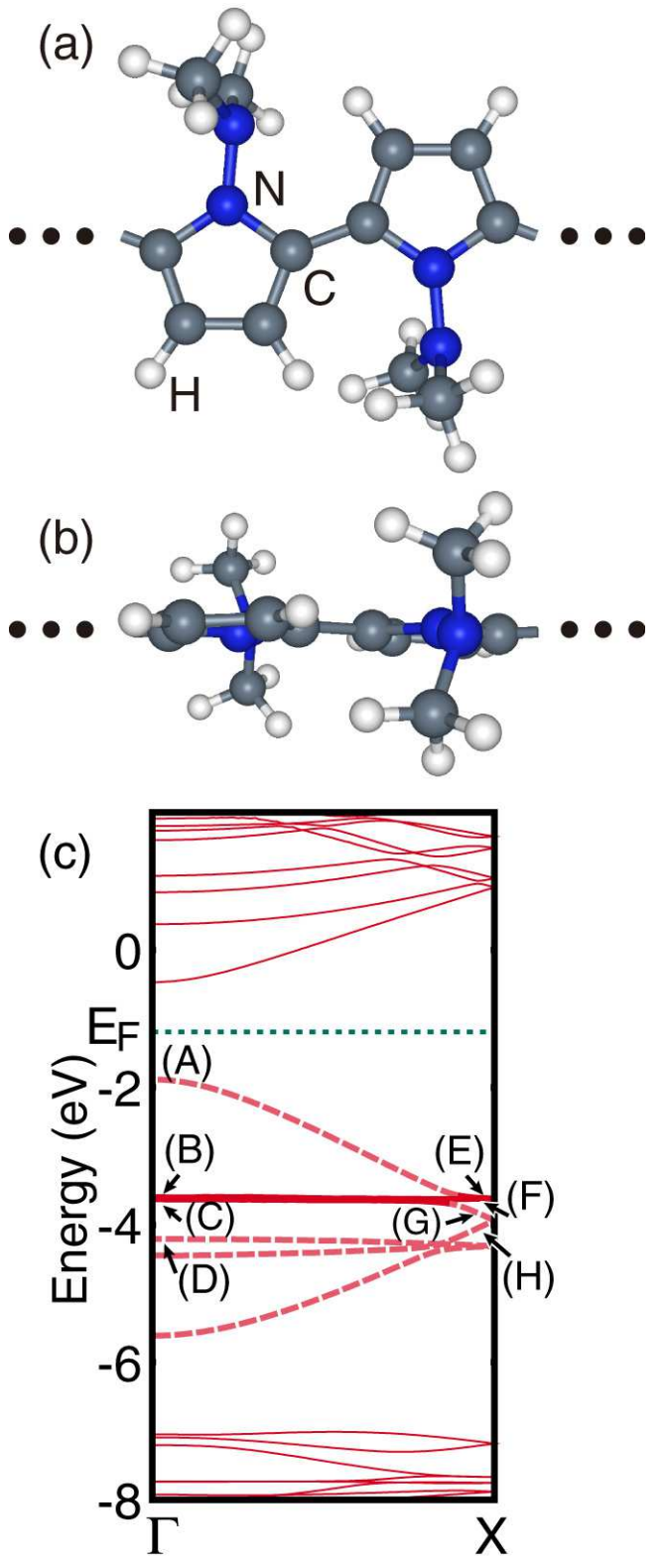

FIG. 1: (Color online) Top view (a) and side view (b) of the optimized atomic structure of polydimethylaminopyrrole in a unit cell. (c) Band structure of undoped polydimethylaminopyrrole. Thick solid (dashed) lines represent $\sigma$-bands ( $\pi$-bands).

band (A)-(G) (uppermost thick dashed line in Fig. 1) as a $\pi$-band.

While in polyaminotriazole $\frac{11,12}{}$ the $\pi$-band is flat, for which the "flat-band ferromagnetism" is expected to be realized, this is no longer the case with polydimethylaminopyrrole, since the $\pi$-band is considerably dispersive. On the other hand the $\sigma$-bands are flat, and one might expect that the flat-band ferromagnetism may occur. This does not apply either, since the bands do not satisfy the local connectivity condition ${ }^{7,8}$ necessary for
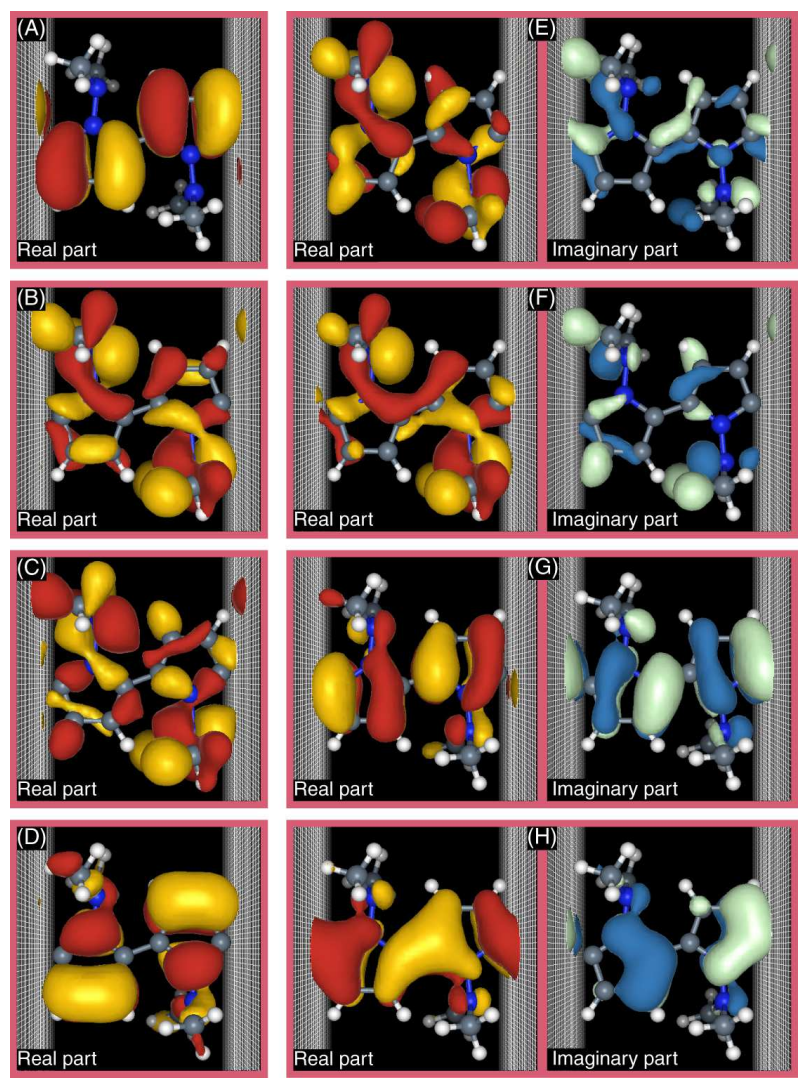

FIG. 2: (Color online) Wave functions in the highest four valence bands of undoped polydimethylaminopyrrole at $\Gamma$ (A-D) or at $\mathrm{X}(\mathrm{E}-\mathrm{H})$ as depicted by isosurfaces with different colors representing the signs of the wave function. See Fig. 1(c) for labels of the energy levels. We have chosen the wave function to be real at $\Gamma$.

the flat-band ferromagnetism. They are flat simply because the transfer between five-membered rings is small, as seen in Figs. 2(B), (C), (E) and (F) where $\sigma$-bonds are absent between those two carbon atoms connecting the adjacent five-membered rings. Although small $\pi$-like orbitals can be seen there, they are negligibly small compared to those of $\pi$-bands in Figs. 2( $\mathrm{G}$ ) and $(\mathrm{H})$.

Does this imply that we have no magnetism? If we perform GGA-SDFT calculations of the hole-doped polydimethylaminopyrrole for various values of hole concentration, spin polarization does appear when the number of holes per unit cell $n_{\mathrm{h}}$ exceeds 1.0. Spins are ferromagnetically ordered or antifrromagnetically ordered depending on the initial condition of the calculation. In the AF state here, up and down spins are aligned alternately in units of a five-membered ring.

Total energies of $\mathrm{F}$ and AF states calculated with GGA-SDFT measured from nonmagnetic (N) states calculated with GGA-DFT are listed in Table \. They are almost the same for each hole concentration within the accuracy of the calculation. Total energies of $\mathrm{F}$ and N states are also nearly the same at $n_{\mathrm{h}}=1.25$ and 1.5 . F and $\mathrm{AF}$ 
TABLE I: Total energies (in eV) of ferromagnetic (F) and antiferromagnetic (AF) states measured from that of nomagnetic (N) state. The values in parenthesis indicate the differences between the numbers of up and down spins per unit cell.

\begin{tabular}{c|c|c|c|c}
\hline$n_{\mathrm{h}}$ & 1.25 & 1.5 & 1.75 & \multicolumn{1}{c}{2.0} \\
\hline \hline polymer & $\mathrm{F}(0.17):+0.008$ & $\mathrm{~F}(0.45):-0.013$ & $\begin{array}{l}\mathrm{F}(0.71):-0.066 \\
\mathrm{AF}:-0.068\end{array}$ & $\begin{array}{l}\mathrm{F}(0.97):-0.145 \\
\mathrm{AF}:-0.152\end{array}$ \\
\hline
\end{tabular}

states could not be obtained even as metastable states when $n_{\mathrm{h}}$ is lower than 1.25 and 1.75 , respectively. These results show that more than half-filled holes $\left(n_{\mathrm{h}} \geq 1.0\right)$ create magnetic instability in this system. In the above calculations, no Peierls instability appeared when geometry optimization is performed for each hole concentration and spin ordering. This fact indicates that the structure of this polymer is quite robust.

Fig. 3 shows the band structures of the hole-doped polydimethylaminopyrrole calculated with GGA-SDFT. While there is no spin polarization for $n_{\mathrm{h}}=0.5$ or 1.0 , spin polarization exists in the $\mathrm{F}$ states at $n_{\mathrm{h}}=1.5$ and 2.0, as indicated in Table 1 .

\section{ORIGIN OF THE MAGNETISM: PERIODIC ANDERSON MODEL}

Next let us consider in detail whether dimethylaminopyrrole can be mapped to the periodic Anderson model, and whether it has a magnetic, especially ferromagnetic ground state. Indeed, here we have narrow $\sigma$ bands embedded in wide $\pi$ bands which hybridize with each other. Theoretically, it has been well known that the periodic Anderson model can have a ferromagnetic ground state in a variety of conditions ${ }^{19}-21$. One famous example is the situation when the model can be mapped to the Kondo lattice model ${ }^{22}$, i.e., when there is one electron in each localized orbital. On the other hand, Batista et al ${ }^{14}$ recently proposed another mechanism which can work when the particle density of the localized state is not close to unity.

The basic idea of Batista et al $\underline{14}$ may be recapitulated as follows. Let us consider the periodic Anderson model whose Hamiltonian is defined as

$$
\begin{aligned}
H= & H_{0}+H_{U} \\
H_{0}= & -t_{c} \sum_{r, r^{\prime}, \sigma}\left(c_{r, \sigma}^{\dagger} c_{r^{\prime}, \sigma}+\text { h.c. }\right) \\
& -t_{f} \sum_{r, r^{\prime}, \sigma}\left(f_{r, \sigma}^{\dagger} f_{r^{\prime}, \sigma}+\text { h.c. }\right)+\varepsilon_{f} \sum_{r, \sigma} n_{r, \sigma}^{f} \\
& +V \sum_{r, \sigma}\left(c_{r, \sigma}^{\dagger} f_{r^{\prime}, \sigma}+\text { h.c. }\right) \\
H_{U}= & -U \sum_{r} n_{r, \uparrow}^{f} n_{r, \downarrow}^{f},
\end{aligned}
$$

where the conduction electrons (created by $c_{r, \sigma}^{\dagger}$ ) hybridize with localized electrons (created by $f_{r, \sigma}^{\dagger}$ ) with $r$ representing the site and $\sigma$ the spin. Here $t_{c}, t_{f}$ are the respective hopping energies, $\varepsilon_{f}$ (assumed to be $\left|\varepsilon_{f}\right|<t_{c}$ ) the energy level of the localized orbital, $V$ the hybridization, and $U$ is the repulsive interaction within the localized orbital. We can diagonalize the one-body part of the two-band Hamiltonian $H_{0}$ to obtain the band representation as

$$
H_{0}=\sum_{k, \sigma}\left(E_{k}^{+} \beta_{k, \sigma}^{\dagger} \beta_{k, \sigma}+E_{k}^{-} \alpha_{k, \sigma}^{\dagger} \alpha_{k, \sigma}\right)
$$

where $E_{k}^{+}>E_{k}^{-}$(see Fig 5 .

Let us introduce two kinds of Wannier orbitals, one dominantly localized,

$$
\phi_{r, \sigma}=\frac{1}{N} \sum_{k}^{K^{>}} e^{\mathrm{i} k r} \beta_{k, \sigma}+\frac{1}{N} \sum_{k}^{K^{<}} e^{\mathrm{i} k r} \alpha_{k, \sigma}
$$

and another dominantly conductive,

$$
\psi_{r, \sigma}=\frac{1}{N} \sum_{k}^{K^{<}} e^{\mathrm{i} k r} \alpha_{k, \sigma}+\frac{1}{N} \sum_{k}^{K^{<}} e^{\mathrm{i} k r} \beta_{k, \sigma}
$$

where we have decomposed the $k$-space into $K^{>}\left(K^{<}\right)$for which the localized (conduction) electrons are the dominant in the $\beta_{k, \sigma}$ band (Fig[5]).

With the new Wannier basis the one-body part of the Hamiltonian now reads

$$
H_{0}=\sum_{r, r^{\prime}} \tau_{r, r^{\prime}}^{\psi} \psi_{r, \sigma}^{\dagger} \psi_{r^{\prime}, \sigma}+\tau_{r, r^{\prime}}^{\phi} \phi_{r, \sigma}^{\dagger} \phi_{r^{\prime}, \sigma}
$$

where $\tau$ 's are new transfer energies with no off-diagonal terms like $\psi_{r, \sigma}^{\dagger} \phi_{r^{\prime}, \sigma}$. Batista et al. ${ }^{14}$ have shown that, while the interaction term in the Hamiltonian becomes complicated in terms of $\psi$ and $\phi$, the dominant term is $U_{\text {eff }} n_{r \sigma}^{\phi} n_{r}^{\phi}$, while the other terms can be neglected when the hybridization $V$ is not too strong. When the system is in the mixed valence regime, namely, when the Fermi energy $E_{F}$ is above $\varepsilon_{f}$ in the present hole-doped case, holes first doubly-occupy the uncorrelated $\psi_{k}$ states which are above $\varepsilon_{f}$, and then go into $\phi_{k}$ states. There $\phi_{k}$ 's tend to have parallel spins, because the cost of the kinetic energy due to the spin polarization $\left(\sim t_{f}\right)$ is small, while the ferromagnetic state does not feel $U_{\text {eff }}$ because of the Pauli's exclusion principle.

When all the $\phi_{k}$ 's are singly occupied (by holes in the present case), we can construct localized $\phi_{r}$ 's by using occupied states only. Because there is no interaction between the spins in this case, the ferromagnetic state and the paramagnetic state have the same energy. When 

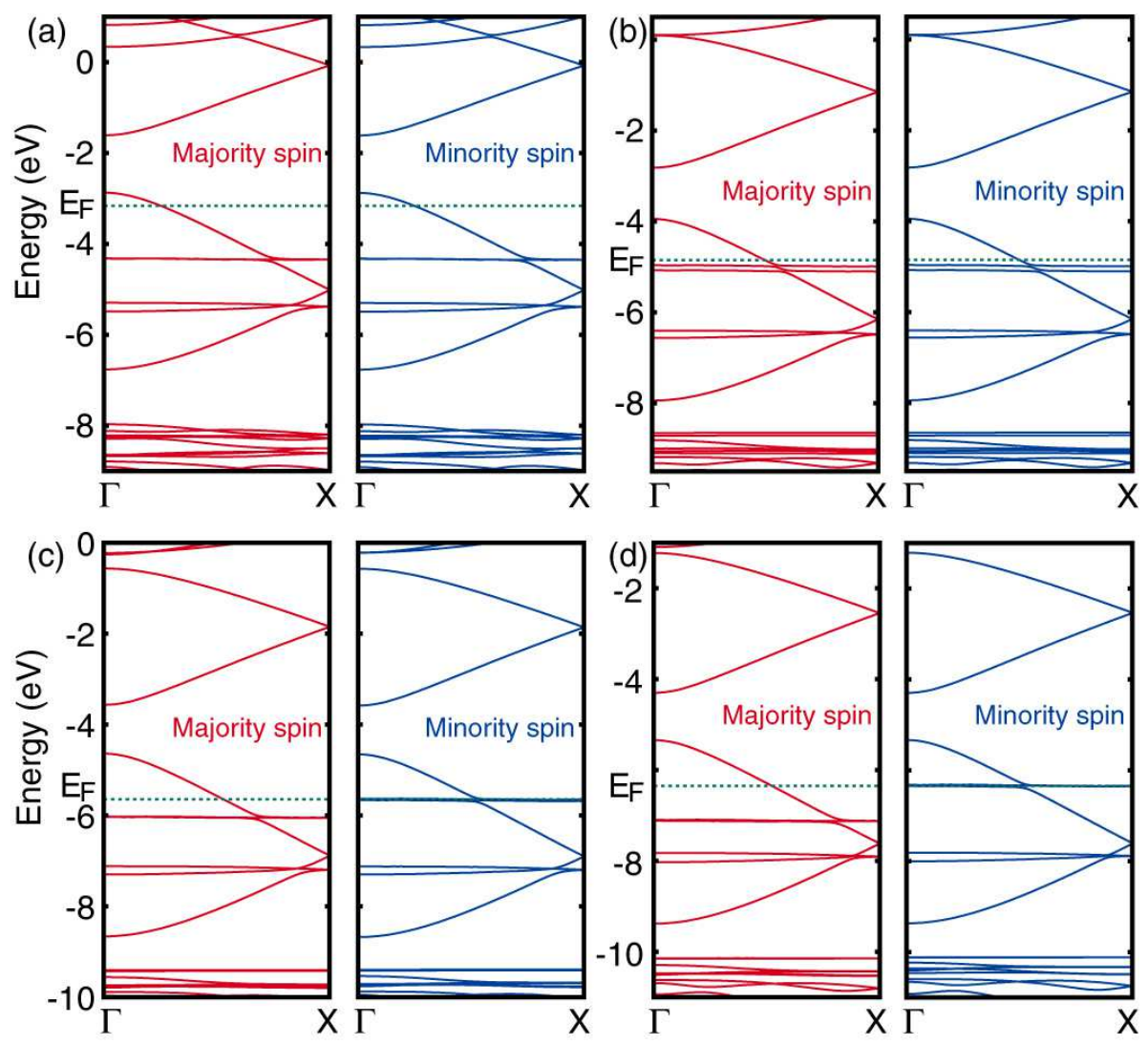

FIG. 3: (Color online) Spin-resolved band structures of the hole-doped polydimethylaminopyrrole for the concentration of holes per unit cell (a) $n_{\mathrm{h}}=0.5$, (b) $n_{\mathrm{h}}=1.0$, (c) $n_{\mathrm{h}}=1.5$ (F state), (d) $n_{\mathrm{h}}=2.0$ (F state).

$\phi_{k}$ 's are only partly occupied, by contrast, we cannot construct completely localized $\phi_{r}$ 's only from the occupied states. Carriers then start to feel $U_{\text {eff }}$ through the overlap of delocalized $\phi_{r}$ 's, which makes the ferromagnetic state energetically favorable. Therefore, the system should choose a ferromagnetic ground state when $\phi_{k}$ states are less than half-filled for $U_{\text {eff }}$ sufficiently greater than $t_{f}$. This has been demonstrated numerically for the one-dimensional periodic Anderson model. ${ }^{14}$

In this mechanism of ferromagnetism, a hybridization between narrow and dispersive bands plays an important role. In polydimethylaminopyrrole, it is clear from the band structures in Fig. 1(c) and Fig 3 (which are summarized in Fig (5) the $\sigma$-band and $\pi$-band are hybridized each other. Although the two bands may seem intersecting, they are actually anticrossing with each other.

\section{TIGHT-BINDING ANALYSIS}

Let us then construct the one-body part of the periodic Anderson Hamiltonian from first principles. The model consists of five sites representing the $\pi$-orbitals in the five-membered ring and an additional "amino N" site. The amino $\mathrm{N}$ site represents the molecular orbital that consists of the $\pi$-orbital of the $\mathrm{N}$ atom in the amino base, the $\sigma\left(\mathrm{sp}^{3}\right)$ orbitals of two methyl bases and $\sigma$-orbitals of the five-membered ring. The transfer and on-site energies shown in the inset of Fig. 4 are fitted to reproduce Fig. 1(c). Obtained values are: $t=2.5 \mathrm{eV}, t_{1}=2.0 \mathrm{eV}$, $\varepsilon_{1}=-1.4 \mathrm{eV}$, and $\varepsilon_{2}=-1.3 \mathrm{eV}$. Figure 4 shows the band structure and wave functions in the tight-binding model. In the tight-binding model the primitive cell contains only one five-membered ring, but we have folded the bands at $\mathrm{X}(k=\pi / 2 a)$ to facilitate comparison with Fig.1(c). We can see that the tight-binding fit successfully reproduces all the relevant (i.e., the thick lines in the DFT result in Fig. $1(\mathrm{c})) \pi$-bands and $\sigma$-band. So the six sites considered in the tight-binding model have turned out to be sufficient for describing the relevant bands (consisting of the mainly $\pi$ bands of the ring, plus another one whose character is not the $\pi$ orbits of the ring). In other words, the bonding between the ring and amino $\mathrm{N}$ can be expressed only by the transfer between the $\pi$ orbital of the $\mathrm{N}$ atom at the apex of the ring (apex $\mathrm{N}$ ) and that at the amino N. Because the molecular orbital that forms $\sigma$-band is included in the amino $\mathrm{N}$ site, the $\sigma$-band of this model has a large amplitude at the amino $N$. In this sense, " $\sigma$-band" (which was meant to mean those other than the $\pi$ orbits of the ring) should rather 


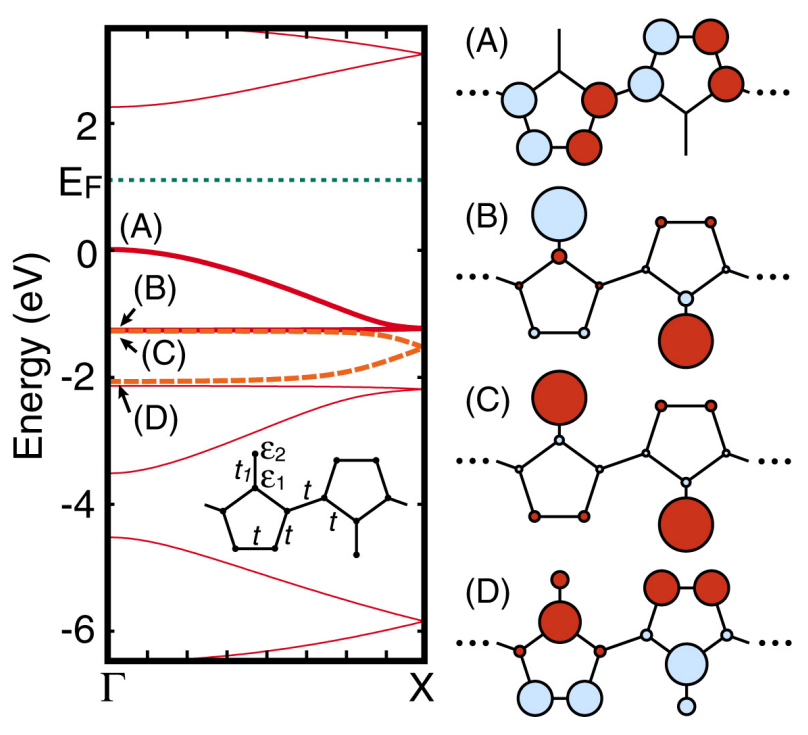

FIG. 4: (Color online) Left: Band structure of polydimethylaminopyrrole in a tight-binding model. Inset shows tightbinding parameters. They are fitted to reproduce Fig. 1(c). Thick solid (dashed) lines correspond to $E_{k}^{+}\left(E_{k}^{-}\right)$in Eq. (4). Right: Wave functions for the highest four occupied states (A-D labeled in the left panel) at $\Gamma$, where the size of each circle represents the amplitude while the different colors its sign.

be called "dimethylamino band", or, put more generally, the functional group band.

The simple model shows that the $\sigma$-band does not satisfy local connectivity condition. The flatness of the band simply comes from localized nature of the wave function. While the tight-binding model here is the same as that for polyaminotriazole ${ }^{11}$ except for the values of parameters, a large difference is that the transfer between the amino $\mathrm{N}$ and apex $\mathrm{N}$ is very small here compared to that in polyaminotriazole, which is precisely because the $\pi$ orbitals of the $\mathrm{N}$ atoms are twisted almost 90 degrees with each other here. The twist also produces hybridization with $\sigma$-orbitals of the five-membered ring. The small transfer between the amino $\mathrm{N}$ and apex $\mathrm{N}$ breaks the subtle balance of parameters necessary to satisfy local connectivity condition for the flat-band ferromagnetism, but simultaneously creates narrow and dispersive bands, mutually hybridized, which is a prerequisite for the ferromagnetism based on the periodic Anderson model.

In order to make the identification of the present band structure with the periodic Anderson model clearer in terms of $\phi$ and $\psi$ states in Eq. (6), we show in Fig. 5 the band structure (Fig. (4) unfolded into an extended Brillouin zone. The upper band (solid line) consisting of a part of the top valence $\pi$-band and a part of the flat $\sigma$-band correspond to $E_{k}^{+}$in Eq. (4), while the lower band (dashed line) consisting of the other parts of $\sigma$ and $\pi$-bands correspond to $E_{k}^{-}$. In the figure, thick lines and thin lines correspond to $\phi$ and $\psi$-states in Eq. (6), re-

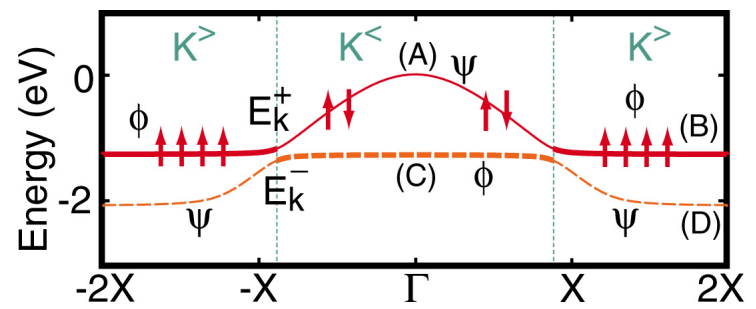

FIG. 5: (Color online) Band structure of polydimethylaminopyrrole in the tight-binding model, unfolded onto an extended Brillouin zone. Solid (dashed) lines correspond to $E_{k}^{+}\left(E_{k}^{-}\right)$in Eq. (4), while thick (thin) lines $\phi(\psi)$ states in Eq. (6) $(\sim \sigma(\pi)$ bands in the present system). Labels (A-D) refer to the band structure in Fig. 4 Arrows schematically indicate the spins of accommodated holes.

spectively. Simply put, $\phi$ represents the $\sigma$-band and $\psi$ the $\pi$-band in the present organic system.

By using obtained tight-binding parameters of the two bands, we performed diagonalization study of periodic Anderson model where each site represents a fivemembered ring and six sites form a ring. Although this model is essentially the same as Ref. [14] except for the values of parameters, obtained ground state was not ferromagnetic state. This result is compatible with the result of GGA-SDFT where total energy of $\mathrm{F}$ state is not definitely lower than that of AF state. This is probably due to the small hybridization $V$ (within the range $V / t_{c}=0.01$ to 0.1 ) compared to that in Ref. [14] (typically $V / t_{c}=0.3$ or 0.5$)$.

In terms of the tight-binding model, we can explain an interesting feature in the GGA-SDFT band structure (Fig. 3) where the energy of the $\sigma$-band shifts relative to the other bands with the doping. For instance, as we increase the number of holes from $n_{\mathrm{h}}=0$ (Fig. 3(a)) to 1.0 (Fig. 3(b)), the $\sigma$-band goes up. When $n_{\mathrm{h}}$ is increased further (Figs. 3(c) and 3(d)), the $\sigma$-band of majority spins starts to go down, while that of minority spins does not. As a result, the lower limit of doping concentration necessary for the spin polarization becomes nearly halffilled $\left(n_{\mathrm{h}}=1.0\right.$, i.e., $1 / 2$ hole per five membered ring). Such a shift of the $\sigma$-band in the GGA-SDFT calculation can be reproduced by using the tight-binding model, if a Coulomb interaction of $1.4 \mathrm{eV}$ between the amino $\mathrm{N}$ site and five-membered ring is assumed and a mean-field approximation is applied. Note that $\pi$ orbital of the amino $\mathrm{N}$ is hybridized with the $\sigma$ orbitals of the five-membered ring, so that the interaction effectively contains an intrasite interaction and can be large.

\section{OLIGOMERS}

We move on to the oligomers of dimethylaminopyrrole. This is practically important because synthesis of the oligomers of dimethylaminopyrrole is possible $\frac{13}{}$ while 


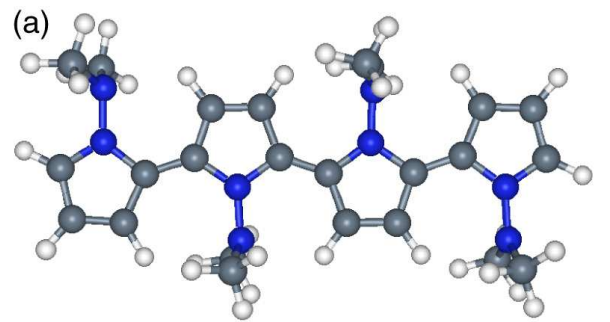

(b)

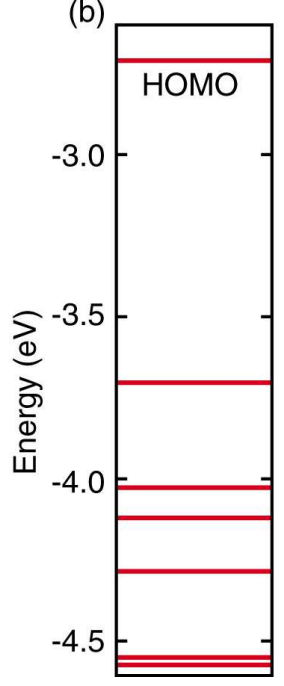

(c) Majority spin Minority spin

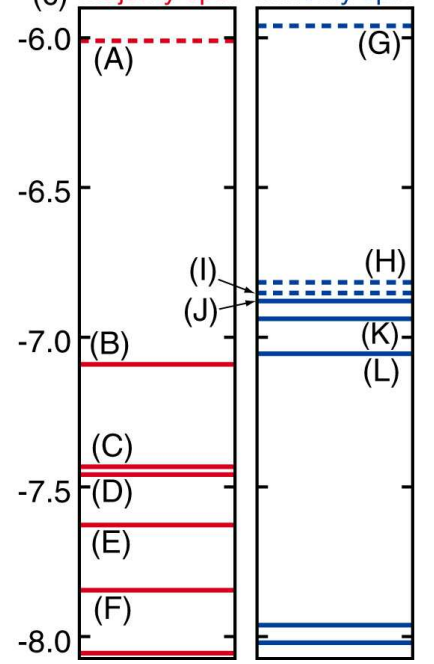

FIG. 6: (Color online) (a) Atomic structure and energy levels of (b) undoped and (c) four-hole-doped (F state) tetramer of dimethylaminopyrrole. Solid (dashed) lines represent occupied (unoccupied) states. Wave functions of the states labeled as (A-L) are shown in Fig. 7 below.

the polymer may be difficult to fabricate. Like the polymer, oligomers of dimethylaminopyrrole have spin polarization if holes are doped enough. Total energies of $\mathrm{F}$ state and AF state are almost the same for each length and number of holes, as will be shown later.

As a typical oligomer, we show the result for a tetramer. Figure 6 shows the atomic structure, the energy levels of undoped nonmagnetic tetramer and fourhole-doped ferromagnetic tetramer. For the ferromagnetic tetramer, we can see in Fig. 6(c) that four consecutive minority-spin states $(\mathrm{H}-\mathrm{K})$ take relatively higher energy levels than corresponding majority-spin states (C$\mathrm{F}$ ), while other levels are almost the same between the majority and minority spins. As a consequence the higher two states out of the four become unoccupied in the minority-spin channel.

The four states are $\sigma$-states, counterparts of the $\sigma$ band in the polymer, which can be confirmed if we look at the wave functions of the four states in Figs. 7(C-F) for majority spin and in Figs. $7(\mathrm{H}-\mathrm{K})$ for minority spin. All these wave functions are seen to consist of nitrogen $\pi$-orbitals and carbon $\sigma$-orbitals, while the other wave functions in Figs. Z (A,B,G,L) consist only of $\pi$-orbitals.

We can relate the arrangement of energy levels of the

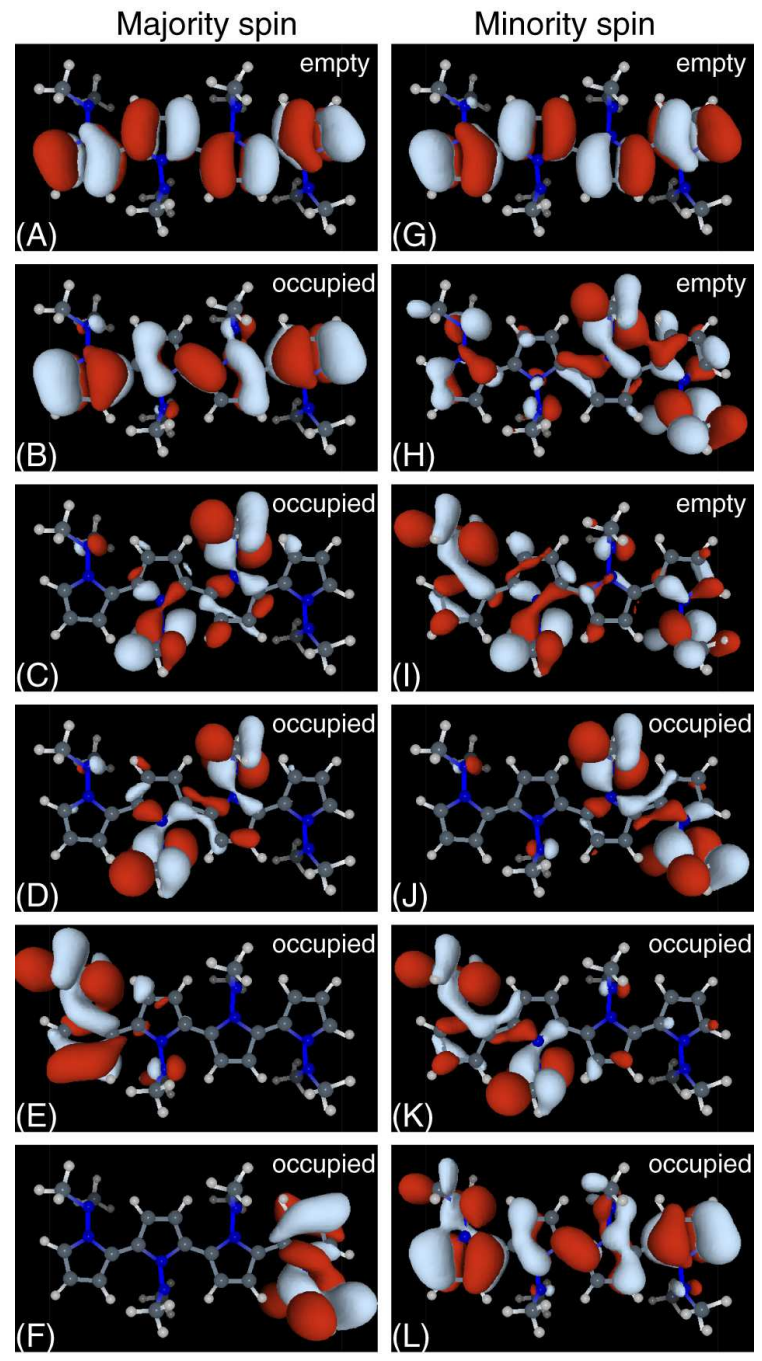

FIG. 7: (Color online) Wave functions of a four-hole-doped tetramer of dimethylaminopyrrole for the energy levels labeled in Fig. 6(c) as depicted by isosurfaces with different colors representing the signs of the wave function.

oligomer with the band structure of the polymer. Generally, for an oligomer consisting of $n$ monomers ( $n$-mer), the number of electronic states constructed from one kind of molecular orbital of the monomer is $n$. Obviously, a state in which all monomers have the same phase is one of the $n$ states, and its wave function is similar to that of the polymer at $\Gamma$-point except for the truncation of the chain. We call it $\Gamma$-state. Another linearly independent state can be constructed by introducing a node into the wave function of the $\Gamma$-state making the signs of the molecular orbitals at the opposite ends reversed. This state is similar to that of the polymer at $k=\pi / n a$ ( $a$ : the length of the monomer) in that the phase rotates by $\pi$ per $n$ monomers. In the same way, $m$-th state of the oligomer corresponding to the polymer's state at $k=(m-1) \pi / n a$ can be constructed by introducing $m-1$ nodes (a phase rotation of $(m-1) \pi)$ in the $\Gamma$-state. Be- 


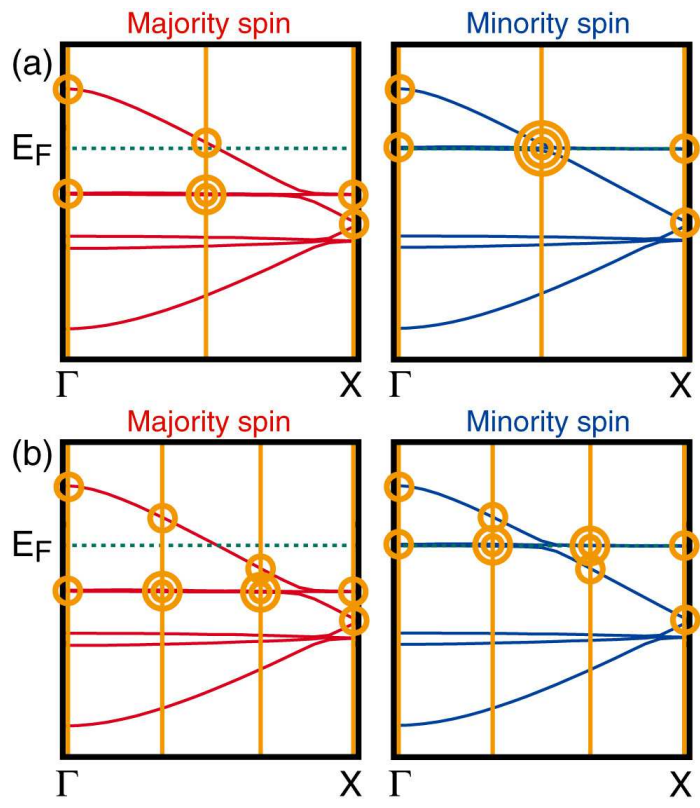

FIG. 8: (Color online) Correspondence between the band structure of the polymer (curves) and the energy levels of oligomers (circles) for dimethylaminopyrrole for the ferromagnetic (a) four-hole-doped tetramer and (b) six-hole-doped hexamer. Single, double, and triple circles indicate one, two and three energy levels in the oligomer, respectively.

cause of the similarity of the wave functions, the energy level of the $m$-th state in $n$-mer can be inferred according to the band energy of the polymer at $k=(m-1) \pi / n a$.

An easy way to do such an estimation is to take the intersecting points of the band with vertical lines dividing the zone in k-space $(k=0$ to $\pi / a)$ into $n$. For example, the energy levels of ferromagnetic four-hole-doped tetramer and six-hole-doped hexamer around the LUMOHOMO can be estimated as in Figs. $8(a)$ and 8 (b), respectively. Note again that the whole band is folded at $\mathrm{X}(k=\pi / 2 a)$ because of two monomers in the unit cell. The band structure of Fig. 3(d) is used here because the hole density of these examples is $n_{\mathrm{h}}=2.0$. We take $n$ to be even in the following for simplicity. As shown in the following, the order of energy levels estimated in this way agrees well with the result for the oligomers. One reason why such an estimation is valid is that the finitesize effect is not very large in the oligomers of dimethylaminopyrrole, probably due to the simple chain structure and not-too-large transfer between monomers.

With such a kind of level scheme, we can estimate how many holes should be doped to realize spin polarization in a given oligomer. For that purpose, whether the vertical line in Fig. 8 exist at $k=\mathrm{X} / 2$ is important, since the $\pi$ band and the flat $\sigma$-band of minority spin cross at this point. When $n / 2$ is odd as in Fig. 8(b), no vertical line exists at X/2 and thus $n / 2+1$ holes first enter the levels coming from the $\pi$-band whose energies are the same for both spins. Since two more holes are necessary to occupy the levels associated with the flat $\sigma$-band, $n / 2+$ 3 holes are necessary in total for the state to be spinpolarized. The total spin in $\mathrm{F}$ state (i.e., the difference of the number of up and down spins, $N_{\uparrow}-N_{\downarrow}$ ) can be estimated to be $N-n / 2-1$, where $N$ is the number of doped holes in an $n$-mer. When $n / 2$ is even, on the other hand, one of the vertical lines passes through $\mathrm{X} / 2$ as in Fig. 8 (a). At $k=X / 2$, the four energy levels, two from the $\pi$-bands for both spins and two from the $\sigma$-bands for the minority spin, almost coincide when holes are doped. Depending on which level is the highest, $n / 2+2$ or $n / 2+4$ holes are necessary to have spin-polarizations in this case, with $N_{\uparrow}-N_{\downarrow}$ estimated to be $N-n / 2$ or $N-n / 2-2$, respectively.

Table [II shows the result of GGA-SDFT calculations for a series of oligomers of dimethylaminopyrrole with various numbers of doped holes. In the calculation of the dimer and tetramer, geometry optimizations are performed for each hole concentration and each spin state, $\mathrm{N}, \mathrm{F}$ and AF. For the hexamer and larger oligomers, geometry optimization is performed only for undoped $\mathrm{N}$ state. Such a treatment is acceptable because there is no significant difference in the optimized structures depending on the spin state and hole concentration in the polymers and small oligomers. The numbers of holes necessary for spin polarization and $N_{\uparrow}-N_{\downarrow}$ of F state almost fully agree with the above estimate in terms of the polymer's band structure. For the $n$-mers with odd $n / 2$, i.e., hexamer and decamer, the values of the spin polarization agree well with the above estimate, which should reflect the fact that no energy levels associated with the $\pi$-band exist near the Fermi energy when spin polarization emerges, as shown in Fig. 8(b). For the oligomers with even $n / 2$, i.e., octamer and dodecamer, $N_{\uparrow}-N_{\downarrow}$ is expected to take either of $N-n / 2$ and $N-n / 2-2$ depending on the order of almost degenerated energy levels of $\pi$-band and $\sigma$-band. The result shows that $N_{\uparrow}-N_{\downarrow}$ is always equal to $N-n / 2$ for all cases. This indicates that the energy level of the $\pi$-band at $\mathrm{X} / 2$ is slightly higher than that of $\sigma$-band.

\section{CONCLUSION}

We have used a first-principles calculation to examine magnetism in a polymer and oligomers of dimethylaminopyrrole. We have found that polydimethylaminopyrrole should have magnetic instability if density of doped holes are more than one hole per unit cell $\left(n_{\mathrm{h}}>1.0\right)$. For $n$-mers of dimethylaminopyrrole, we conclude that $n / 2+2$ or $n / 2+3$ holes are necessary to have spin-polarization depending on whether $n / 2$ is even or odd, respectively. While polydimethylaminopyrrole belongs to the same category (chains of five-membered rings) as the previously proposed polyaminotriazole $e^{9.11}$ which has theoretically shown to realize the flat-band

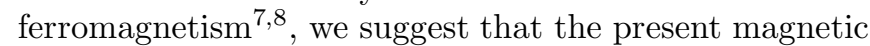
instability can be related to the mechanism proposed by 
TABLE II: Total energies (in eV) of $\mathrm{F}$ and AF states measured from $\mathrm{N}$ state are shown for various oligomers (rows) with various numbers of holes (columns). Boxes marked with $\mathrm{N}$ denote that the total energies of $\mathrm{F}$ and AF states are higher than that of $\mathrm{N}$ state or do not give converged results. The values in parenthesis indicate $N_{\uparrow}-N_{\downarrow}$ for F state.

\begin{tabular}{|c|c|c|c|c|c|c|}
\hline & 2 holes & 4 holes & 6 holes & 8 holes & 10 holes & 12 holes \\
\hline 2 -mer & $\mathrm{N}$ & & & & & \\
\hline 4 -mer & $\mathrm{N}$ & $\begin{array}{l}F(2.00):-0.18 \\
\text { AF:- } 0.20\end{array}$ & & & & \\
\hline 6 -mer & $\mathrm{N}$ & $\mathrm{N}$ & $\begin{array}{l}F(2.24):-0.20 \\
A F:-0.19\end{array}$ & & & \\
\hline 8-mer & $\mathrm{N}$ & $\mathrm{N}$ & $\begin{array}{l}F(1.84):-0.07 \\
A F:-0.06\end{array}$ & $\begin{array}{l}\mathrm{F}(3.89):-0.39 \\
\mathrm{AF}:-0.36\end{array}$ & & \\
\hline 10-mer & $\mathrm{N}$ & $\mathrm{N}$ & $\mathrm{N}$ & $\begin{array}{l}F(2.63):-0.08 \\
A F:-0.08\end{array}$ & $\begin{array}{l}F(4.11):-0.51 \\
A F:-0.50\end{array}$ & \\
\hline 12-mer & $\mathrm{N}$ & $\mathrm{N}$ & $\mathrm{N}$ & $\begin{array}{l}\mathrm{F}(1.84):-0.01 \\
\mathrm{AF}: 0.00\end{array}$ & $\begin{array}{l}\mathrm{F}(3.70):-0.21 \\
\mathrm{AF}:-0.22\end{array}$ & $\begin{array}{l}\mathrm{F}(5.55):-0.57 \\
\mathrm{AF}:-0.56\end{array}$ \\
\hline
\end{tabular}

Batista et al $\underline{14}$ based on the periodic Anderson model.

Realization of ferromagnetism is not yet sufficient, because the total energies of the ferromagnetic state and antiferromagnetic state is almost degenerate in GGA-SDFT calculations. Diagonalizations of the model Hamiltonians constructed from the band structure do not show ferromagnetic ground states, either. Since this comes from the smallness of the hybridization $V$ between the flat band and the dispersive band, we can conversely predict that ferromagnetism will be stabilized if we can increase $V$. In the present material $V$ is determined mainly by $\pi$ bonding between the nitrogen atom in the amino base and the nitrogen atom in the five membered ring. In dimethylaminopyrrole, $V$ is very small because the angle between the two $\pi$-orbitals is nearly 90 degrees. Therefore, $V$ can be increased if this angle is decreased by replacing dimethylamino base with another base. Note that reducing the angle to 0 would be excessive because that is the same as the case of aminotriazole whose electronic state applies to the mechanism of flat-band ferromagnetism rather than periodic Anderson model.

So the present work gives an interesting case of material design where control of the parameters of electronic states has lead to a different mechanism for the many- body effects, namely organic periodic Anderson model and associated ferromagnetism. An important future problem is whether the SDFT calculations can capture the ferromagnetism à la Batista. Another future problem is how the present concept of the organic periodic Anderson model can be extended to wider materials beyond those considered here. Studying inter-oligomer couplings will also be important for examining ferromagnetic materials. As for the doping the material, we can consider various chemical dopants as discussed for in Ref. [10].

\section{Acknowledgments}

We would like to thank Hiroshi Nishihara, Yoshinori Yamanoi and Norikazu Ohshima for extensive discussions on the chemistry of polymers and oligomers, and also for the collaboration in fabricating the materials. This work was partly supported by a Grant-in-Aid for Scientific Research from Japan Society for the Promotion of Science. The first-principles calculations were performed with TAPP (the Tokyo Ab-initio Program Package).
1 P.-M. Allemand, K. C. Khemani, A. Koch, F. Wudl, K. Holczer, S. Donovan, G. Gruner and J. D. Thompso, Science 253, 301 (1991).

2 A. Rajca, J. Wongsriratanakul, and S. Rajca, Science 294, 1503 (2001).

3 E. C. Pereira et al. J. Magn. Magn. Mater. 226-230, 2023 (2001).

4 A. A. Correa et al. Synth. Met. 121, 1836 (2001).

5 O. R. Nascimento et al., Phys. Rev. B 67, 144422 (2003).

${ }^{6}$ F. R. de Paula et al., J. Magn. Magn. Mater. 320, e193 (2008).

7 A. Mielke and H. Tasaki, Commun. Math. Phys. 158, 341 (1993)

8 H. Tasaki, Prog. Thoer. Phys. 99, 489 (1998).
9 R. Arita, Y. Suwa, K. Kuroki, and H. Aoki, Phys. Rev. Lett. 88, 127202 (2002).

${ }^{10}$ R. Arita, Y. Suwa, K. Kuroki, and H. Aoki, Phys. Rev. B 68140403 (2003).

11 Y. Suwa, R. Arita, K. Kuroki, and H. Aoki, Phys. Rev. B 68, 174419 (2003).

12 Y. Suwa, R. Arita, K. Kuroki, and H. Aoki, e-J. Surf. Sci. Nanotech. 2, 38 (2004).

13 Y. Yamanoi, T. Hamada, K. Ohshima, and H. Nishihara, unpublished.

14 C. D. Batista, J. Bonča, and J. E. Gubernatis, Phys. Rev. B 68, 214430 (2003).

15 J. P. Perdew, K. Burke, and Y. Wang, Phys. Rev. B 54, 16533 (1996). 
16 J. A. White and D. M. Bird, Phys. Rev. B 50, 4954 (1994).

17 D. Vanderbilt, Phys. Rev. B 41, 7892 (1990).

18 K. Laasonen, A. Pasquarello, R. Car, C. Lee, and D. Vanderbilt, Phys. Rev. B 47, 10142 (1993).

19 Z. Gulácsi and D. Vollhardt, Phys. Rev. Lett. 91186401 (2003)

20 Z. Gulácsi and D. Vollhardt, Phys. Rev. B 72, 075130
(2005)

21 Z. Gulácsi, A. Kampf, and D. Vollhardt, Prog. Theor. Phys. Suppl. 176, 1 (2008).

${ }^{22}$ H. Tsunetsugu, M. Sigrist, and K. Ueda, Rev. Mod. Phys. 69, 809 (1997). 